Editorial

\title{
El impacto de las Ciencias de la Computación en el mundo real
}

\section{The impact of Computer Science in the real world}

\author{
Gloria Piedad Gasca-Hurtado ${ }^{1}$, Liliana Machuca-Villegas ${ }^{1,2}$ \\ gpgasca@udem.edu.co, liliana.machuca@correounivalle.edu.co \\ ${ }^{1}$ Universidad de Medellín, Carrera 87 No. 30-65, 50026, Medellín, Colombia \\ ${ }^{2}$ Universidad del Valle, Calle 13 \# 100-oo, 760032, Cali, Valle del Cauca, Colombia \\ DOI: 10.17013/risti.29.o
}

\section{Introducción}

Los artículos presentados en este número fueron seleccionados de los trabajos aceptados en el Undécimo Seminario de Ciencias de la Computación (SICC) 2018, realizado en la Ciudad de Medellín, Antioquia, Colombia durante los días 10, 11 y 12 de octubre del 2018. Este seminario ha tenido una tasa de aceptación del 13.63\% de los artículos enviados.

En este número de la Revista Ibérica de Sistemas y Tecnologías de la Información (RISTI), se abordan temas relacionados con las "Ciencias de la Computación". Los trabajos de este número son las versiones originales presentadas por los autores en comunicación oral durante el congreso SICC 2018 y que fueron seleccionados después de realizarse un proceso de evaluación por pares doble ciego.

\section{Ciencias de la computación}

El impacto de las Ciencias de la Computación en la vida y el desarrollo de la humanidad es invaluable, sus desarrollos como disciplina han hecho realidad muchas ideas que parecían imposibles (Pulla, Limaye, \& Yajnik, 2016) años atrás. Tal vez, por esta razón es que actualmente las Ciencias de la Computación es una disciplina reconocida por su aporte a la humanidad más que por sus aportes en computación o informática, como posiblemente se pensó en sus inicios (Denning, Tedre, \& Yongpradit, 2017). 
El impacto que tiene la solución de problemas reales a través de las Ciencias de la Computación y el aporte que constantemente hace al desarrollo de la vida, la reconocen como una disciplina que deja de lado el diseño y la construcción de máquinas o robots, para constituirse en una ciencia preocupada por la construcción de herramientas que solucionen problemas de la humanidad. Por ejemplo, los lenguajes de programación son una herramienta esencial y central de la disciplina, que facilita la implementación de soluciones en el mundo real, ya sea de índole académica o científica, en áreas de la ciencia como la medicina, la biología, la informática o la computación.

En consecuencia, las Ciencias de la computación es una disciplina base para el desarrollo de las otras disciplinas con una gama de posibilidades amplia, desde sus fundamentos teóricos y algorítmicos hasta desarrollos de vanguardia en robótica, visión artificial, sistemas inteligentes, bioinformática entre otras áreas. Se extiende desde la teoría hasta la programación.

Como parte del aporte de las Ciencias de la Computación en educación, existe una preocupación constante porque, desde edades tempranas, se enseñe a programar independientemente de las preferencias profesionales a futuro. Por lo anterior, adquirir conocimiento adecuado es indispensable y por ende es necesario que las instituciones educativas tengan herramientas para mejorar los ambientes de aprendizaje, los cuales comúnmente dependen de condiciones biológicas, psicológicas, ambientales, escolares y de comunidad (Gómez, Aduna, García, Cisneros, \& Padilla, 2004).

Por otro lado, el impacto de las Ciencias de la Computación en términos de soluciones para la sociedad se ve reflejado con el avance informático en términos de desarrollo de software, en especial en el ámbito de nuevas herramientas para acelerar los procesos de desarrollo de software. En este sentido, la calidad es el atributo que tiene mayor relevancia en la determinación del éxito de un producto software (Puello, Feliu, \& CalvoManzano, 2016).

Asociado a la calidad del software, se ve reflejado también en las Ciencias de la Computación la mejora de procesos, con el objetivo de analizar y definir cómo mejorar las prácticas de desarrollo software, partiendo de una evaluación del proceso en uso. En este esquema de mejora de proceso software los factores humanos se consideran también factores críticos de éxito asociados a los procesos de desarrollo de software (Casañola, Estrada, \& León-Rodríguez, 2014).

Sin embargo, no solo los factores críticos de éxito son materia de investigación en esta disciplina. Los enfoques de calidad de software trascienden modelos y/o estándares como guías para lograr que productos de software respondan a las necesidades de la sociedad y la industria.

Siendo hoy en día las empresas pequeñas o medianas las de mayor presencia en la industria del software en cualquier país, existen propuestas orientadas a cubrir necesidades de estas empresas en la implementación de buenas prácticas de ingeniería de software para el desarrollo de productos de calidad que les permitan mejorar su operación y sus procesos (Sanchez-Gordon, de Amescua, O’Connor, \& Larrucea, 2017) (Larrucea, O'Connor, Colomo-Palacios, \& Laporte, 2016). 
Por otro lado, las nuevas tecnologías de la sociedad de la información inmersas también en la disciplina Ciencias de la Computación están formando parte directa de las profundas transformaciones sociales a las que se viene asistiendo en las últimas décadas (Atienza, 2017). Dentro de los servicios de distribución de contenidos multimedia, se destaca el servicio de video bajo demanda VoD (Pripužić et al., 2013) que cuenta ventajas importantes. Sin embargo, ciertos problemas que dificultan la interacción del usuario han sido identificados en el marco de investigaciones que pretenden generar propuestas a dichos problemas con el fin de facilitar el acceso ágil al contenido multimedia.

Finalmente, en el marco de las Ciencias de la Computación con una relevante importancia se avanza en el desarrollo de modelos computacionales que interrelacionados con experimentación permiten el diseño de novedosas estrategias de control en el campo de la automatización, la robótica y la manufactura.

Las aristas de esta disciplina que se han mencionado son parte de los avances investigativos que se presentan en los trabajos seleccionados para ser publicados en este número de RISTI. Estos trabajos han sido seleccionados porque abordan temáticas de interés en la actualidad y se enmarcan en soluciones del mundo real con un enfoque asociado a las Ciencias de la Computación.

\section{Estructura}

En el primer artículo se realiza un estudio sobre estilos de aprendizaje aplicando técnicas de minería de datos a la información derivada de la aplicación de dos test llamados VARK y CHAEA a los estudiantes de la Institución Educativa Joaquín Cárdenas Gómez del Municipio de San Carlos - Antioquia (Colombia). Como resultado de este estudio, se genera un plan de apoyo a la gestión directiva con la identificación de factores que afectan el aprendizaje. Entre los hallazgos más relevantes está la verificación de que los estilos de aprendizaje por sí solos no influyen en la repitencia, se requiere la combinación de otros elementos como la edad y el uso de varios estilos de aprendizaje.

En el segundo artículo se propone una guía que facilita la aplicación del modelo de DeLone \& McLean, denominado D\&M, para la evaluación de productos de software para pequeños entornos. El principal aporte de esta guía radica en la definición de pasos y en el catálogo de preguntas preestablecidas para que un pequeño entorno, sin poseer conocimientos acabados en el modelo, pueda rápidamente preparar los instrumentos necesarios para su aplicación. Esta guía fue aplicada en una empresa, demostrando su utilidad para ayudar a los pequeños entornos en la evaluación de los productos de software que dan soporte a sus procesos de negocio.

El tercer artículo tiene como objetivo proponer una primera versión de la estructura de un catálogo de factores humanos, identificados a partir de un conjunto de factores críticos de éxitos los cuales afectan en la mejora de procesos de software en una organización. La principal motivación de este trabajo está dada por la cantidad de empresas de software que continuamente buscan mejorar los procesos necesarios para la creación de un producto de software. 
El cuarto artículo es el reporte de una experiencia de la implementación del estándar ISO/IEC 29110 para reforzar el proceso de desarrollo de software de cuatro centros de desarrollo de software de universidades mexicanas. El objetivo de este reporte es aportar facilidades a las organizaciones donde se evidencia una creciente necesidad de mejorar sus procesos de desarrollo de software, con el fin de desarrollar productos y servicios de calidad. Para lograrlo, dichas organizaciones deben cumplir dos requisitos: (1) integrar una cultura de procesos y mejora continua en las organizaciones, y (2) dotar de personal altamente cualificado que tenga el conocimiento y habilidades para trabajar exitosamente con modelos y/o estándares utilizados en las organizaciones. Estos requisitos son abordados desde la experiencia en el trabajo presentado.

El quinto artículo presenta la identificación de algunas debilidades en el servicio de video bajo demanda (VoD), que han sido detectadas a pesar de su amplia difusión. Específicamente, en el trabajo se propone una arquitectura basada en contexto para el servicio de $\mathrm{VoD}$, la cual considera las diferentes dimensiones del contexto y es organizada en 4 vistas: de negocio, de contexto, funcional y de implementación. Esta arquitectura pretende enriquecer la funcionalidad del servicio y contribuir con dos de los problemas identificados del servicio de VoD.

Finalmente, el sexto artículo presenta una estrategia de control novedosa para resolver el problema de regulación de un sistema de servo posicionamiento neumático. En el trabajo se presentan y discuten los resultados de simulaciones y experimentos con un cilindro neumático sin vástago, de $25 \mathrm{~mm}$ de diámetro y una válvula de control proporcional de 5/3 vías. Dentro de los hallazgos más importantes está el desempeño en posicionamiento y respuestas simétricas en el movimiento del deslizador en ambas direcciones.

\section{Agradecimientos}

Agradecemos al director de la Revista Álvaro Rocha por otorgar la oportunidad para dar a conocer los trabajos más relevantes presentados en el seminario SICC 2018. Asimismo, expresamos nuestra gratitud a los autores de los trabajos publicados, lo cual hizo posible este número en RISTI.

\section{Referencias}

Atienza, C. N. (2017). El video bajo demanda en televisión: test de causalidad Granger en los ciclos de vida de un servicio video bajo demanda. Madrid: Universidad Complutense de Madrid.

Casañola, Y. T., Estrada, C. A. F., \& León-Rodríguez, G. (2014). Modelo Si. MPS. CU para valorar las organizaciones al iniciar la mejora de proceso de software. Revista Cubana de Ciencias Informáticas, 8, 92-103.

Denning, P. J., Tedre, M., \& Yongpradit, P. (2017). Misconceptions about computer science. Communications of the ACM, 6o(3), 31-33. 
Gómez, L., Aduna, A., García, E., Cisneros, A., \& Padilla, J. (2004). Manual de estilos de aprendizaje. Material Autoinstruccional Para Docentes y Orientadores Educativos (pp. 22-29). México: Secretaría de Educación Pública. Subsecretaría de Educación Media Superior. Dirección General Del Bachillerato. Dirección de Coordinación Académica.

Larrucea, X., O'Connor, R. V, Colomo-Palacios, R., \& Laporte, C. Y. (2016). Software process improvement in very small organizations. IEEE Software, 33(2), 85-89.

Pripužić, K., Žarko, I. P., Podobnik, V., Lovrek, I., Čavka, M., Petković, I., ... Gojčeta, M. (2013). Building an IPTV VoD recommender system: An experience report. In 12th International Conference on Telecommunications (ConTEL), 2013 (pp. 155-162).

Puello, J. de J. J., Feliu, T. S., \& Calvo-Manzano, J. A. (2016). Una aproximación basada en metamodelado del área de proceso de Validación del CMMI: Un caso de estudio. RISTI-Revista Ibérica de Sistemas e Tecnologias de Informação, (17), 26-40.

Pulla, N., Limaye, U., \& Yajnik, S. (2016). Can you find a way to define Computer Science? Revista Antioqueña de Las Ciencias Computacionales, 6(2).

Sanchez-Gordon, M.-L., de Amescua, A., O'Connor, R. V, \& Larrucea, X. (2017). A standard-based framework to integrate software work in small settings. Computer Standards \& Interfaces, 54, 162-175. 